\title{
VI. Entrenamiento muscular inspiratorio en el paciente con enfermedad pulmonar obstructiva crónica
}

GRAÇA PINHEIRO DE C.* y FERNANDO SALDÍAS P.*

$\underline{\text { Inspiratory muscle training in patients with chronic obstructive pulmonary disease }}$

Respiratory muscle weakness is observed in chronic obstructive pulmonary disease (COPD) patients and contributes to hypoxemia, hypercapnia, dyspnoea, nocturnal oxygen desaturation and reduced exercise performance. During exercise it has been shown that diaphragm work is increased in COPD and COPD patients use a larger proportion of the maximal inspiratory pressure (PImax) than healthy subjects. This pattern of breathing is closely related to the dyspnoea sensation during exercise and might potentially induce respiratory muscle fatigue. However, diaphragmatic fatigue was not demonstrated after exhaustive exercise. Studies in patients with COPD have shown natural adaptations of the diaphragm to greater oxidative capacity and resistance to fatigue. The above mentioned considerations gave conflicting arguments to the rationale of inspiratory muscle training (IMT) in COPD. Both IMT alone and IMT as adjunct to general exercise reconditioning significantly increased inspiratory muscle strength and endurance. A significant effect was found for dyspnoea at rest and during exercise. Improved functional exercise capacity tended to be an additional effect of IMT alone and as an adjunct to general exercise reconditioning, but this trend did not reach statistical significance. No significant correlations were found for training effects with patient characteristics. However, subgroup analysis in IMT plus exercise training revealed that patients with inspiratory muscle weakness improved significantly more compared to patients without inspiratory muscle weakness. From this review it is concluded that inspiratory muscle training is an important addition to a pulmonary rehabilitation programme directed at chronic obstructive pulmonary disease patients with inspiratory muscle weakness. The effect on exercise performance is still to be determined. In summary, IMT improves inspiratory muscle strength and endurance, functional exercise capacity, dyspnoea and quality of life. Inspiratory muscle endurance training was shown to be less effective than respiratory muscle strength training.

Key words: Chronic obstructive pulmonary disease; dyspnoea; exercise capacity; muscle training; respiratory muscles; inspiratory muscle strength; inspiratory muscle training.

\section{Resumen}

Los pacientes con enfermedad pulmonar obstructiva crónica (EPOC) tienen debilidad de los músculos respiratorios, lo cual contribuye a la disnea, hipoxemia, hipercapnia, desaturación nocturna y limitación de la actividad física. Durante el ejercicio se ha demostrado que aumenta el trabajo del diafragma en pacientes con EPOC y utilizan una mayor proporción de la presión inspiratoria máxima (PImax) comparado con los sujetos sanos. El patrón respiratorio anormal de los pacientes con EPOC está relacionado con la sensación de disnea durante el ejercicio y, potencialmente, podría inducir a la fatiga muscular respiratoria. Sin embargo, la fatiga del diafragma no se ha demostrado después de un ejercicio intenso. Los estudios en pacientes con EPOC han demostrado cambios adaptativos en las fibras musculares del diafragma que tienen mayor capacidad oxidativa y resistencia a la fatiga. De este modo, existen argumentos contradictorios en relación al beneficio clínico obtenido con el entrenamiento de los músculos inspiratorios (EMI) en pacientes con EPOC. El EMI aislado o como complemento de ejercicios de reacondicionamiento general aumenta significativamente la fuerza muscular inspiratoria y la resistencia a la fatiga, disminuyendo significativamente la disnea en reposo y durante el ejercicio. Además, los estudios sugieren que el EMI tiende a mejorar la capacidad funcional para realizar ejerci-

* Departamento de Enfermedades Respiratorias, Facultad de Medicina, Pontificia Universidad Católica de Chile. 
cio, efecto favorable que no alcanzó significación estadística. El análisis de subgrupos ha demostrado mayor beneficio clínico del EMI en los pacientes con debilidad muscular inspiratoria. De esta revisión se concluye que el entrenamiento muscular inspiratorio puede ser útil en pacientes seleccionados con enfermedad pulmonar obstructiva crónica, que tienen disfunción muscular inspiratoria comprobada, insertado en un programa de rehabilitación integral. El efecto sobre la capacidad de realizar ejercicio aún no ha sido determinado. En resumen, el EMI aumenta la fuerza muscular inspiratoria y la resistencia a la fatiga, mejorando la capacidad funcional, la disnea y la calidad de vida de los pacientes con EPOC.

Palabras clave: Enfermedad pulmonar obstructiva crónica; disnea; capacidad de ejercicio; entrenamiento muscular; músculos respiratorios; fuerza de músculos inspiratorios; entrenamiento muscular inspiratorio.

\section{Introducción}

Los pacientes con enfermedad pulmonar obstructiva crónica (EPOC) presentan limitación crónica del flujo aéreo que no revierte con el uso de broncodilatadores asociado a debilidad de los músculos respiratorios y periféricos de magnitud variable ${ }^{1}$. La disfunción de la musculatura esquelética ha sido atribuida a múltiples factores tales como la hipoxia e hipercapnia crónica, inflamación sistémica, estrés oxidativo, desnutrición, desacondicionamiento físico y otros factores relacionados con las comorbilidades asociadas ${ }^{2-8}$. La interacción de los distintos factores que actúan tanto a nivel local como sistémico sobre el músculo esquelético ocasionaría disminución de la fuerza y resistencia a la fatiga que afecta de forma heterogénea a los distintos grupos musculares $^{9}$. La mayoría de los pacientes con EPOC tiene debilidad de la musculatura inspiratoria ${ }^{10,11}$, siendo el diafragma el principal músculo comprometido, en conjunto con la musculatura intercostal, tal alteración aparece en etapas precoces de la enfermedad ${ }^{9,12}$.

El diafragma de los pacientes con EPOC se adapta a la sobrecarga crónica y aumenta la resistencia a la fatiga ${ }^{4,10}$. A un mismo volumen pulmonar, los músculos inspiratorios son capaces de generar más fuerza comparado con sujetos controles sanos. Esto ocurre precozmente en el curso de la enfermedad, incluso antes que se produzcan adaptaciones en el músculo esquelético ${ }^{10,11}$. En comparación con el cuádriceps, el diafragma es relativamente resistente a la fatiga con el ejercicio de alta intensidad y la ventilación voluntaria máxima en pacientes con EPOC, y las fibras musculares aisladas del diafragma serían metabólicamente más eficientes comparado con controles normales. Sin embargo, estos pacientes a menudo tienen hiperinflación, lo cual coloca a sus músculos inspiratorios en desventaja mecánica.

La hiperinsuflación es un fenómeno característico de la EPOC, que está presente en reposo y aumenta durante el ejercicio (hiperinflación dinámica) asociado a los cambios en el patrón respiratorio frente a la mayor demanda metabólica. $\mathrm{La}$ hiperinflación dinámica ocasiona aplanamiento del diafragma, acortamiento de las fibras musculares y pérdida de la zona de aposición muscular en el tórax, dejando a los músculos inspiratorios en desventaja mecánica, disminuyendo la fuerza y resistencia a la fatiga del diafragma, principal músculo inspiratorio, lo cual tiene implicancias en la génesis de la disnea de los pacientes con EPOC y la limitación de la capacidad de ejercicio $^{13,14}$.

La disfunción de la musculatura inspiratoria estaría también determinada por anomalías estructurales en los músculos inspiratorios (reducción de las fibras oxidativas y enzimas glicolíticas) ${ }^{4-8}$, pero se ha documentado mediante biopsias del diafragma de los pacientes con EPOC, que este músculo presentaría cambios estructurales que le permitirían adaptarse a las desventajas mecánicas y mejorar su función ${ }^{15-17}$. La estructura de los músculos inspiratorios y espiratorios puede ser modificada por el entrenamiento muscular específico, por eso se postula que el entrenamiento muscular inspiratorio (EMI) permitiría mejorar la fuerza y resistencia a la fatiga de los músculos respiratorios ${ }^{9}$. Cuando la carga del entrenamiento muscular es adecuada, los ejercicios de los músculos inspiratorios alivian la disnea y aumentan la capacidad de realizar actividad física9.

\section{Preguntas}

1. ¿El entrenamiento muscular inspiratorio disminuye la disnea y aumenta la capacidad de realizar actividad física en pacientes con EPOC?

2. ¿Quiénes se beneficiarían con el entrenamiento muscular inspiratorio?

3. ¿Qué técnicas de entrenamiento muscular inspiratorio han demostrado ser efectivas?

4. ¿Está indicado el entrenamiento muscular espiratorio en pacientes con EPOC? 


\section{Pacientes}

Pacientes con enfermedad pulmonar obstructiva crónica estable que presentan disnea y limitación de la capacidad de realizar ejercicio a pesar del tratamiento farmacológico óptimo.

\section{Intervención}

Entrenamiento muscular inspiratorio en pacientes con enfermedad pulmonar obstructiva crónica.

\section{Objetivo}

Evaluar si el EMI alivia la disnea, aumenta la capacidad de realizar ejercicio y mejora la calidad de vida en los pacientes con EPOC.

\section{Resumen de la evidencia}

Los estudios clínicos de entrenamiento de los músculos inspiratorios han sido realizados en atletas, pacientes con enfermedades respiratorias crónicas (asma, fibrosis quística, EPOC), insuficiencia cardíaca congestiva, daño espinal cervical crónico y distrofia muscular, antes de cirugía cardíaca y en la desconexión de ventilación mecánica.

En la Tabla 1 se describen los principales estudios controlados que han examinado los efectos fisiológicos y beneficios clínicos del entrenamiento muscular inspiratorio en pacientes con enfermedad pulmonar obstructiva crónica ${ }^{18-40}$. Los estudios clínicos controlados de EMI han sido de calidad metodológica variable en términos de tamaño muestral, randomización, descripción de las características de la población, pérdida de sujetos en el seguimiento, medición ciega de los resultados y análisis de los resultados por intención de tratar. Las principales variables fisiológicas y clínicas examinadas fueron los cambios en la fuerza muscular inspiratoria, resistencia a la fatiga muscular inspiratoria, función pulmonar, capacidad de ejercicio, disnea y calidad de vida.

En Chile, los estudios de Lisboa y cols, demostraron que el EMI realizado en el domicilio con una válvula de carga umbral con una presión inspiratoria correspondiente al 30\% de la presión inspiratoria máxima (PIMax) aumentaba la fuerza muscular inspiratoria, disminuía la disnea y mejoraba el patrón ventilatorio de los pacientes con EPOC durante el ejercicio ${ }^{29,30}$. Además, el EMI aumentó la distancia recorrida en la prueba de caminata de 6 minutos con una menor demanda metabólica para un ejercicio de similar magnitud.

En la guía clínica norteamericana de rehabilitación pulmonar ${ }^{41}$ se incluyeron seis estudios clínicos controlados que evaluaron la eficacia del EMI en pacientes con EPOC $29,31-33,35,36$, los cuales eran randomizados, emplearon una carga apropiada de entrenamiento muscular y midieron los efectos fisiológicos (fuerza y tolerancia a la fatiga de los músculos respiratorios) y clínicos (disnea y estado de salud) del EMI. Los estudios clínicos examinados demostraron mejoría significativa de la función muscular inspiratoria, aumento de la capacidad de ejercicio y reducción de la disnea. Estos hallazgos coinciden con los resultados del metanálisis realizado por Lötters y $\operatorname{cols}^{42}$, quienes señalaron que el EMI aumentaba la fuerza y resistencia a la fatiga de los músculos inspiratorios en pacientes con EPOC, y mejoraba significativamente la disnea relacionada a actividades de la vida diaria y durante el ejercicio. El panel de expertos concluyó que la evidencia científica aún era insuficiente y no permitía sustentar el uso rutinario del EMI en los programas de rehabilitación pulmonar (nivel de recomendación 1B). Basados en esta información, el EMI debería ser considerado en pacientes seleccionados con EPOC, quienes tuvieran debilidad muscular inspiratoria, disnea y limitación de la capacidad de realizar ejercicio a pesar de recibir tratamiento farmacológico óptimo.

En el metanálisis realizado por Lötters y cols ${ }^{42}$, incluyeron quince estudios clínicos controlados que evaluaron la eficacia del EMI en pacientes con EPOC ${ }^{18-31,35}$, demostrando beneficios en la fuerza y tolerancia a la fatiga de los músculos inspiratorios y disminución de la disnea de reposo y durante el ejercicio. El análisis de subgrupos demostró que los efectos beneficiosos del EMI se observaron exclusivamente en los pacientes que presentaban debilidad muscular inspiratoria $\left(\mathrm{PIMax} \leq 60 \mathrm{~cm} \mathrm{H}_{2} \mathrm{O}\right)^{21,23,25,28,31,35}$. Además, se observó una tendencia a la mejoría de la capacidad de realizar ejercicio en los pacientes entrenados, la cual no alcanzó significación estadística.

Geddes y $\operatorname{cols}^{43}$, en una revisión sistemática incluyeron 16 estudios clínicos controlados que examinaron los efectos del EMI en pacientes con EPOC $^{19,20,22,24,27,29-32,34-40}$, los principales hallazgos fueron: a) El EMI aumentó la fuerza de los músculos inspiratorios, el flujo inspiratorio máximo y la resistencia a la fatiga de los músculos respiratorios en pacientes con EPOC; b) El EMI aumentó la capacidad de realizar ejercicio y disminuyó la intensidad de la disnea durante el ejercicio; c) El EMI mejoró la calidad de vida en pacientes con EPOC comparado con el grupo control.

O'Brien y $\operatorname{cols}^{44}$, realizaron una revisión sistemática de los estudios clínicos controlados que compararon el efecto del entrenamiento muscular 


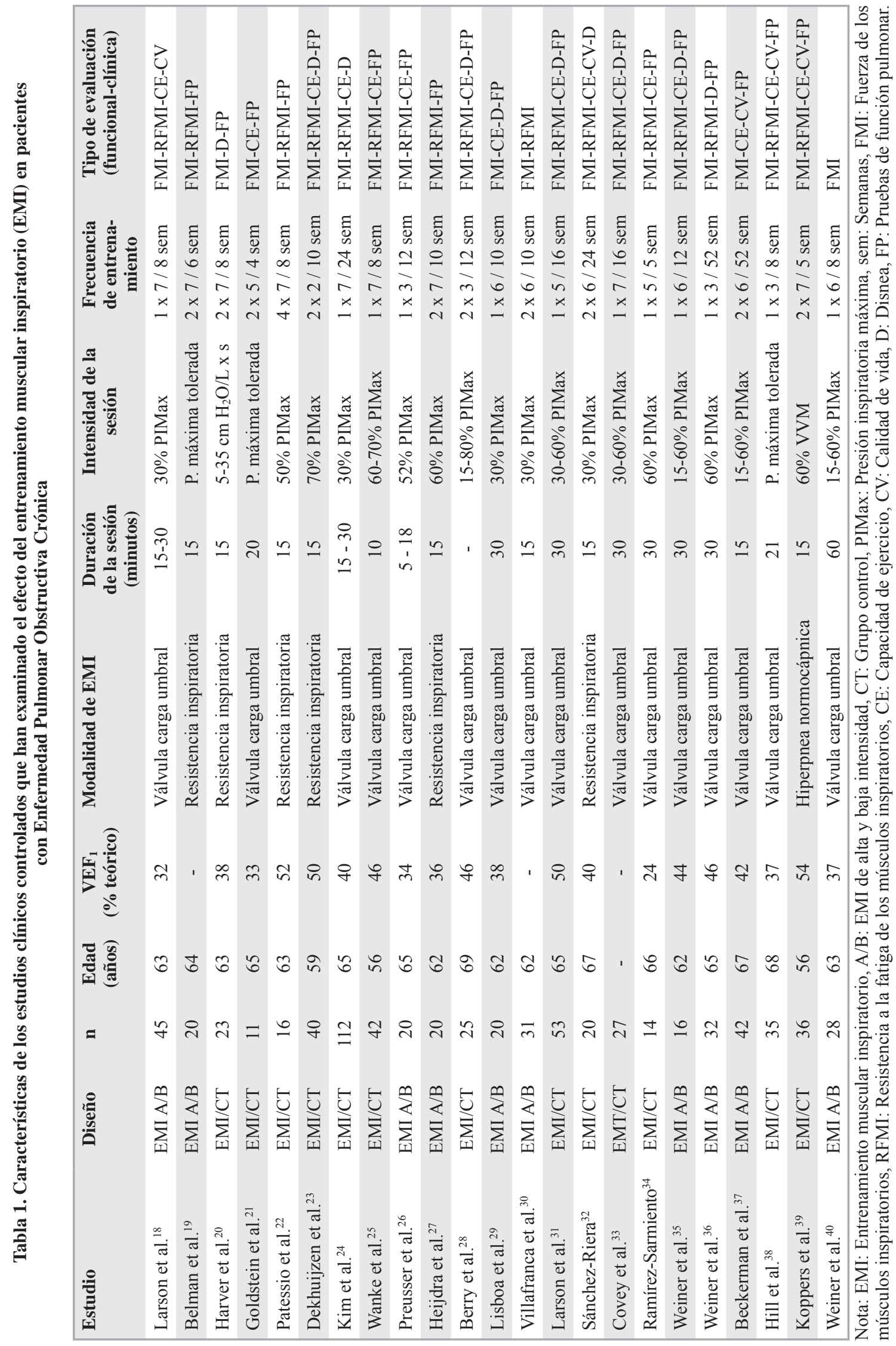


inspiratorio aislado o asociado a un programa de ejercicios con un programa de ejercicios regular. Los autores incluyeron 18 estudios clínicos controlados y los principales hallazgos fueron que hubo una mejoría significativa de la fuerza muscular inspiratoria y el volumen corriente durante el ejercicio máximo en los pacientes que recibieron EMI asociado al programa regular de ejercicio.

Weiner y $\operatorname{cols}^{36}$, demostraron los beneficios a largo plazo del EMI en pacientes con EPOC avanzado, obteniendo mejoría significativa de la performance muscular (fuerza y resistencia), capacidad funcional y alivio de la disnea que se mantuvo durante un año en un programa de rehabilitación pulmonar (EMI con válvula de carga umbral ajustada a $60 \%$ de la PIMax). Mientras que en el grupo control, tal beneficio no se pudo mantener cuando el entrenamiento fue suspendido. Güell y cols ${ }^{45}$ y Troosters y cols ${ }^{46}$, también demostraron beneficio del EMI que se mantuvo a lo largo de un año de entrenamiento. Después de una etapa breve de entrenamiento supervisado por el equipo de salud, los autores demostraron que los pacientes eran capaces de realizar los ejercicios en su domicilio, logrando buena adherencia al programa a un costo razonable ${ }^{45,46}$.

Heijdra y $\operatorname{cols}^{27}$, obtuvieron un aumento significativo de la saturación nocturna de oxígeno en pacientes con EPOC avanzado entrenados con válvula tipo umbral (EMI con 60\% PIMax) durante 10 semanas, lo cual se asoció a aumento de la fuerza y resistencia muscular diafragmática y de la musculatura intercostal.

El entrenamiento muscular inspiratorio puede realizarse empleando diferentes técnicas que buscan mejorar la fuerza y resistencia a la fatiga de los músculos respiratorios ${ }^{12,41}$. En los primeros estudios de entrenamiento muscular respiratorio se emplearon diferentes técnicas tales como la hiperpnea voluntaria isocápnica que involucra la musculatura inspiratoria y espiratoria, la cual es actualmente utilizada en estudios de investigación. En el EMI se pueden utilizar dispositivos de resistencia inspiratoria y válvulas de carga umbral. Las principales ventajas de los dispositivos de carga umbral son el costo accesible, aprendizaje simple de la técnica y que las presiones de trabajo se logran de manera independiente del flujo inspiratorio. El efecto beneficioso del EMI en pacientes con EPOC ha sido demostrado empleando los diferentes dispositivos, siendo lo más importante que se aplique la carga de entrenamiento apropiada ${ }^{41-43}$. El análisis de subgrupos empleando dispositivos de resistencia inspiratoria ${ }^{19,20,22,23,27}$ y válvulas de carga umbral ${ }^{18,21,24-26,29-31,35-38,40}$ demostró cambios similares en la fuerza y resistencia a la fatiga de los músculos inspiratorios ${ }^{42}$.

Los programas de entrenamiento muscular inspiratorio pueden ser realizados en el domicilio, utilizando válvula umbral o de resistencia ${ }^{41-43}$. Los programas son de bajo costo pero requieren supervisión y los beneficios funcionales se pierden rápidamente al suspender el programa de entrenamiento ${ }^{36,45,46}$. En un editorial, McConnel apoya el EMI como una medida de tratamiento eficaz, destacando que se trata de una intervención de bajo costo y sin efectos adversos ${ }^{47}$. Las principales contraindicaciones del EMI son la insuficiencia respiratoria aguda $\left(\mathrm{PaO}_{2}<60 \mathrm{mmHg}\right.$ y $\mathrm{PaCO}_{2}>45 \mathrm{mmHg}$ respirando aire ambiente), hipertensión pulmonar severa, enfermedades cardiovasculares e hipertensión arterial crónica descompensadas y la fatiga muscular respiratoria ${ }^{9,12}$.

Se han propuesto diferentes protocolos de $\mathrm{EMI}^{18-40}$, pero la intensidad, frecuencia y duración ideal de los ejercicios aún no han sido establecidas mediante estudios clínicos controlados y randomizados, existiendo una gran variedad de prescripciones sugeridas. En términos generales ${ }^{9,12}$, se propone realizar sesiones de EMI de 15-30 minutos, con una frecuencia de entrenamiento de 1-2 veces al día, durante 3-5 días de la semana, midiendo los cambios de la PIMax cada 4-5 semanas, durante un período de tiempo indefinido. Las presiones programadas en la válvula umbral deberían variar entre el $30-70 \%$ de la presión inspiratoria máxima medida para cada paciente.

La musculatura espiratoria tiene vital importancia en el reflejo de la tos y el esfuerzo de la tos eficaz, la cual puede estar disminuida en pacientes con EPOC. Se ha comunicado que la fuerza y resistencia a la fatiga de los músculos espiratorios estarían disminuidas en pacientes con EPOC avanzado $^{14,48,49}$. Los estudios de entrenamiento muscular espiratorio han sido escasos ${ }^{35,50}$, los cuales sugieren que existiría un probable beneficio funcional y clínico (aumento de la fuerza y tolerancia a la fatiga de los músculos espiratorios, y aumento de la capacidad de ejercicio). Sin embargo, el entrenamiento muscular espiratorio no aportaría beneficios clínicos significativos al paciente con $\mathrm{EMI}^{35,51}$, por lo cual no ha sido considerado en forma rutinaria en los programas de rehabilitación respiratoria ${ }^{4,9,12}$.

\section{Grado de recomendación}

El entrenamiento muscular inspiratorio puede ser útil en pacientes seleccionados con enfermedad pulmonar obstructiva crónica, que tienen 
disfunción muscular inspiratoria comprobada, insertado en un programa de rehabilitación integral (calidad de la evidencia B, fuerza de la recomendación moderada). Los programas de entrenamiento muscular inspiratorio pueden ser realizados en el domicilio, utilizando válvula umbral o de resistencia, ambos sistemas han demostrado ser eficaces (calidad de la evidencia B, fuerza de la recomendación moderada).

\section{Bibliografía}

1.- GOSSELINK R, TROOSTERS T, DECRAMER M. Distribution of muscle weakness in patients with stable chronic obstructive pulmonary disease. J Cardiopulm Rehabil 2000; 20: 353-60.

2.- BERNARD S, LEBLANC P, WHITTOM F, CARRIER G, JOBIN J, BELLEAU R, et al. Peripheral muscle weakness in patients with chronic obstructive pulmonary disease. Am J Respir Crit Care Med 1998; 158: 629-34.

3.- SERRES I, GAUTIER V, VARRAY A, PRÉFAUT C. Impaired skeletal muscle endurance related to physical inactivity and altered lung function in COPD patients. Chest 1998; 113: 900-5.

4.- AMERICAN THORACIC SOCIETY/EUROPEAN RESPIRATORY SOCIETY. Skeletal muscle dysfunction in chronic obstructive pulmonary disease: a statement of the American Thoracic Society and European Respiratory Society. Am J Respir Crit Care Med 1999; 159: S1-S40.

5.- GOSKER H R, VAN MAMEREN H, VAN DIJK P J, ENGELEN M P, VAN DER VUSSE G J, WOUTERS E F, et al. Skeletal muscle fibre-type shifting and metabolic profile in patients with chronic obstructive pulmonary disease. Eur Respir J 2002; 19: 617-25.

6.- COUILLARD A, MALTAIS F, SAEY D, DEBIGARÉ $\mathrm{R}$, MICHAUD A, KOECHLIN C, et al. Exerciseinduced quadriceps oxidative stress and peripheral muscle dysfunction in patients with chronic obstructive pulmonary disease. Am J Respir Crit Care Med 2003; 167: 1664-9.

7.- DEBIGARÉ R, MARQUIS K, CÔTE C H, TREMBLAY R R, MICHAUD A, LEBLANC $P$, et al. Catabolic/anabolic balance and muscle wasting in patients with COPD. Chest 2003; 124: 83-9.

8.- ALLAIRE J, MALTAIS F, DOYON JF, NOËL M, LEBLANC P, CARRIER G, et al. Peripheral muscle endurance and the oxidative profile of the quadriceps in patients with COPD. Thorax 2004; 59: 673-8.

9.- MOTA CASALS S. Entrenamiento de los músculos respiratorios en la enfermedad pulmonar obstructiva crónica. En: Guell R, de Lucas Pilar, eds. Tratado de rehabilitación respiratoria. Barcelona: Ars Medica 2005; 221-30.

10.- BÉGIN P, GRASSINO A. Inspiratory muscle dys- function and chronic hypercapnia in chronic obstructive pulmonary disease. Am Rev Respir Dis 1991; 143: 90512.

11.- POLKEY M I, KYROUSSIS D, HAMNEGARD C H, MILLS G H, GREEN M, MOXHAM J. Diaphragm strength in chronic obstructive pulmonary disease. Am J Respir Crit Care Med 1996; 154: 1310-7.

12.- PLEGUEZUELOS COBO E. Rehabilitación de la musculatura respiratoria. En: Pleguezuelos E, Miranda G, Gómez A, Capellas L, eds. Rehabilitación Integral en el Paciente con Enfermedad Pulmonar Obstructiva Crónica. Madrid: Editorial Médica Panamericana 2008; 134-145.

13.- SIMILOWSKI T, YAN S, GAUTHIER A P, MACKLEM P T, BELLEMARE F. Contractile properties of the human diaphragm during chronic hyperinflation. $\mathrm{N}$ Engl J Med 1991; 325: 917-23.

14.- ROCHESTER D F, BRAUN N M. Determinants of maximal inspiratory pressure in chronic obstructive pulmonary disease. Am Rev Respir Dis 1985; 132: $42-7$.

15.- POLKEY M I, KYROUSSIS D, HAMNEGARD C H, MILLS G H, HUGHES P D, GREEN M, et al. Diaphragm performance during maximal voluntary ventilation in chronic obstructive pulmonary disease. Am J Respir Crit Care Med 1997; 155: 642-8.

16.- OTTENHEIJM C A, HEUNKS L M, SIECK G C, ZHAN W Z, JANSEN S M, DEGENS H, et al. Diaphragm dysfunction in chronic obstructive pulmonary disease. Am J Respir Crit Care Med 2005; 172: 200-5.

17.- STUBBINGS A K, MOORE A J, DUSMET M, GOLDSTRAW P, WEST T G, POLKEY M I, et al. Physiological properties of human diaphragm muscle fibres and the effect of chronic obstructive pulmonary disease. J Physiol 2008; 586: 2637-50.

18.- LARSON J L, KIM M J, SHARP J T, LARSON D A. Inspiratory muscle training with a pressure threshold breathing device in patients with chronic obstructive pulmonary disease. Am Rev Respir Dis 1988; 138: 68996.

19.- BELMAN M J, SHADMEHR R. Targeted resistive ventilatory muscle training in chronic obstructive pulmonary disease. J Appl Physiol 1988; 65: 2726-35.

20.- HARVER A, MAHLER D A, DAUBENSPECK J A. Targeted inspiratory muscle training improves respiratory muscle function and reduces dyspnea in patients with chronic obstructive pulmonary disease. Ann Intern Med 1989; 111: 117-24.

21.- GOLDSTEIN R, DE ROSIE J, LONG S, DOLMAGE T, AVENDANO M A. Applicability of a threshold loading device for inspiratory muscle testing and training in patients with COPD. Chest 1989; 96: 564-71.

22.- PATESSIO A, RAMPULLA C, FRACCHIA C, IOLI F, MAJANI U, DE MARCHI A, et al. Relationship between the perception of breathlessness and inspiratory resistive loading: report on a clinical trial. Eur Respir J 1989; 7 (Suppl): S587-91. 
23.- DEKHUIJZEN P N, FOLGERING H T, VAN HERWAARDEN C L. Target-flow inspiratory muscle training during pulmonary rehabilitation in patients with COPD. Chest 1991; 99: 128-33.

24.- KIM M J, LARSON J L, COVEY M K, VITALO C A, ALEX C G, PATEL M. Inspiratory muscle training in patients with chronic obstructive pulmonary disease. Nurs Res 1993; 42: 356-62.

25.- WANKE T, FORMANEK D, LAHRMANN H, BRATH H, WILD M, WAGNER C, et al. Effects of combined inspiratory muscle and cycle ergometer training on exercise performance in patients with COPD. Eur Respir J 1994; 7: 2205-11.

26.- PREUSSER B A, WINNINGHAM M L, CLANTON T L. High- vs low-intensity inspiratory muscle interval training in patients with COPD. Chest 1994; 106: 110-7.

27.- HEIJDRA Y F, DEKHUIJZEN P N, VAN HERWAARDEN C L, FOLGERING H T. Nocturnal saturation improves by target-flow inspiratory muscle training in patients with COPD. Am J Respir Crit Care Med 1996; 153: 260-5.

28.- BERRY M J, ADAIR N E, SEVENSKY K S, QUINBY A, LEVER H M. Inspiratory muscle training and wholebody reconditioning in chronic obstructive pulmonary disease. Am J Respir Crit Care Med 1996; 153: 1812-6.

29.- LISBOA C, VILLAFRANCA C, LEIVA A, CRUZ E, PERTUZÉ J, BORZONE G. Inspiratory muscle training in chronic airflow limitation: effect on exercise performance. Eur Respir J 1997; 10: 537-42.

30.- VILLAFRANCA C, BORZONE G, LEIVAA, LISBOA C. Effect of inspiratory muscle training with an intermediate load on inspiratory power output in COPD. Eur Respir J 1998; 11: 28-33.

31.- LARSON J L, COVEY M K, WIRTZ S E, BERRY J K, ALEX C G, LANGBEIN W E, et al. Cycle ergometer and inspiratory muscle training in chronic obstructive pulmonary disease. Am J Respir Crit Care Med 1999; 160: 500-7.

32.- SÁNCHEZ RIERA H, MONTEMAYOR RUBIO T, ORTEGA RUIZ F, CEJUDO RAMOS P, DEL CASTILLO OTERO D, ELÍAS HERNÁNDEZ T, et al. Inspiratory muscle training in patients with COPD: effect on dyspnea, exercise performance, and quality of life. Chest 2001; 120: 748-56.

33.- COVEY M K, LARSON J L, WIRTZ S E, BERRY J K, POGUE N J, ALEX C G, et al. High-intensity inspiratory muscle training in patients with chronic obstructive pulmonary disease and severely reduced function. J Cardiopulm Rehabil 2001; 21: 231-40.

34.- RAMÍREZ-SARMIENTO A, OROZCO-LEVI M, GUELL R, BARREIRO E, HERNÁNDEZ N, MOTA S, et al. Inspiratory muscle training in patients with chronic obstructive pulmonary disease: structural adaptation and physiologic outcomes. Am J Respir Crit Care Med 2002; 166: 1491-7.

35.- WEINER P, MAGADLE R, BECKERMAN M, WEI-
NER M, BERAR-YANAY N. Comparison of specific expiratory, inspiratory, and combined muscle training programs in COPD. Chest 2003; 124: 1357-64.

36.- WEINER P, MAGADLE R, BECKERMAN M, WEINER M, BERAR-YANAY N. Maintenance of inspiratory muscle training in COPD patients: one year follow-up. Eur Respir J 2004; 23: 61-5.

37.- BECKERMAN M, MAGADLE R, WEINER M, WEINER P. The effects of 1 year of specific inspiratory muscle training in patients with COPD. Chest 2005; 128: 3177-82.

38.- HILL K, JENKINS S C, PHILIPPE D L, CECINS N, SHEPHERD K L, GREEN D J, et al. High-intensity inspiratory muscle training in COPD. Eur Respir J 2006; 27: 1119-28.

39.- KOPPERS R J, VOS P J, BOOT C R, FOLGERING $\mathrm{H}$ T. Exercise performance improves in patients with COPD due to respiratory muscle endurance training. Chest 2006; 129: 886-92.

40.- WEINER P, WEINER M. Inspiratory muscle training may increase peak inspiratory flow in chronic obstructive pulmonary disease. Respiration 2006; 73: 151-6.

41.- RIES A L, BAULDOFF G S, CARLIN B W, CASABURI R, EMERY C F, MAHLER D A, et al. Pulmonary Rehabilitation: Joint ACCP/AACVPR Evidence-Based Clinical Practice Guidelines. Chest 2007; 131 (5 Suppl): 4S-42S.

42.- LÖTTERS F, VAN TOL B, KWAKKEL G, GOSSELINK R. Effects of controlled inspiratory muscle training in patients with COPD: a meta-analysis. Eur Respir J 2002; 20: 570-6.

43.- GEDDES E L, O'BRIEN K, REID W D, BROOKS D, CROWE J. Inspiratory muscle training in adults with chronic obstructive pulmonary disease: an update of a systematic review. Respir Med 2008; 102: 1715-29.

44.- O'BRIEN K, GEDDES E L, REID W D, BROOKS D, CROWE J. Inspiratory muscle training compared with other rehabilitation interventions in chronic obstructive pulmonary disease: a systematic review update. J Cardiopulm Rehabil Prev 2008; 28: 128-41.

45.- GÜELL R, CASAN P, BELDA J, SANGENIS M, MORANTE F, GUYATT G H, et al. Long-term effects of outpatients rehabilitation of COPD: a randomized trial. Chest 2000; 117: 976-83.

46.- TROOSTERS T, GOSSELINK R, DECRAMER M. Short- and long- term effects of outpatient rehabilitation in patients with chronic obstructive pulmonary disease: a randomized trial. Am J Med 2000; 109: 207-12.

47.- MCCONNELL A K, WEINER P, ROMER L M. Inspiratory muscular training as a tool for the management of patients with COPD. Eur Respir J 2004; 24: 510-1.

48.- CROPP A, DIMARCO A F. Effects of intermittent negative pressure ventilation on respiratory muscle function in patients with severe chronic obstructive pulmonary disease. Am Rev Respir Dis 1987; 135: 1056-61.

49.- RAMÍREZ-SARMIENTO A, OROZCO-LEVI M, BA- 
RREIRO E, MÉNDEZ R, FERRER A, BROQUETAS

$\mathrm{J}$, et al. Expiratory muscle endurance in chronic obstructive pulmonary disease. Thorax 2002; 57: 132-6.

50. WEINER P, MAGADLE R, BECKERMAN M, WEINER M, BERAR-YANAY N. Specific expiratory mus- cle training in COPD. Chest 2003; 124: 468-73.

51. WEINER P, MCCONELL A. Respiratory muscle training in chronic obstructive pulmonary disease: inspiratory, expiratory, or both? Curr Opin Pulm Med 2005; 11: 140-4.

Correspondencia a:

Dr. Graça Pinheiro de C.

Departamento de Enfermedades Respiratorias,

Facultad de Medicina,

Pontificia Universidad Católica de Chile.

E-mail: graca_pinheiro@hotmail.com 